\title{
ANALYSIS OF OPERATIONAL PROPERTIES OF VOIP NETWORK
}

\author{
Cyril Filip KOVÁČIK, Gabriel BUGÁR \\ Department of Electronics Multimedia Telecommunications, Faculty of Electrical Engineering and Informatics, Technical University \\ of Košice, Letná 9, 04200 Košice, E-mail: cyril.filip.kovacik@student.tuke.sk, gabriel.bugar@tuke.sk
}

\begin{abstract}
Voice transmission over the Internet network is now taken for granted. Many end-user applications address this issue. However, this paper focuses on the specific use of the SCCP protocol created by Cisco, its implementation in a computer network and end devices, determination of the operational properties of this implementation, and their comparison in different conditions. VoIP traffic is compared at different bandwidths and implemented by different configurations of IP protocols. By investigated implementations of IP protocols are meant IPv4, IPv6, and IPv4 protocol with applied NAT. As part of the application of various IP protocols is also compared VoIP communication with a video stream on a local basis. The conclusion of the paper is devoted to the graphical evaluation of these observations and to draw conclusions based on them.
\end{abstract}

Keywords: VoIP, SCCP protocol, IPv4, NAT, IPv6, Wireshark

\section{INTRODUCTION}

The Internet has overcome a major technological leap since its inception. The initial fragments of the Internet were put together as separate university networks. Later were followed by the ARPANET project, which can be considered the direct predecessor of the Internet. Thanks to the ARPANET project in 1969, a descriptive model of the operation of the IEEE 7498 network, better known as the ISO / OSI model, was also created. This model splits the network's service into seven layers, each of which guarantees the network's functionality while being unchanged by the others. The model is effective as an abstract model in terms of the division of tasks between the layers themselves. The TCP / IP protocol, based on the ISO / OSI model, was developed due to its high time intensity. [1] It retains the functions of the original seven layers but combines them in its own four layers. This protocol is now standard in most computer networks. Within the ISO / OSI model, the most important to the research was the network layer, the 3rd layer of this model. This layer contains packet headers containing the version of the IP protocol used and all the information needed for addressing the IP protocol used. The length differential between IPv4 and IPv6 addresses is significant, and as seen in Fig. 1, this difference enhances the length of the packet header. [7] This discrepancy leads to the fact that a longer packet header length may result in slower packet processing.

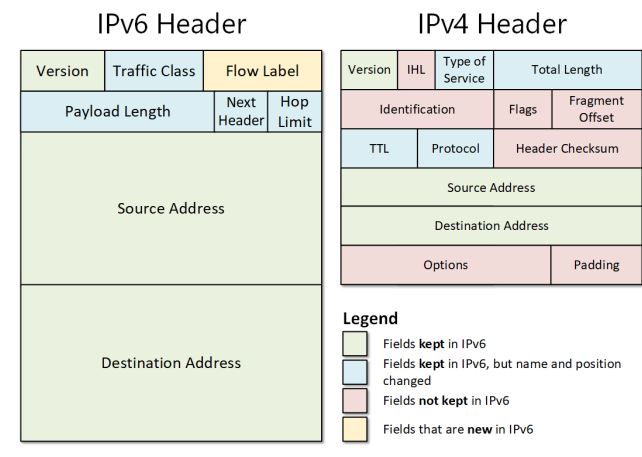

Fig. 1 Comparation of Max Jitter between VoIP and videostream.
This difference led to the conclusion that a longer packet header length could cause extra time processing the packets themselves. By implementing NAT, we have added another step to IPv4 addressing that the router must perform, so it was appropriate to examine this implementation variant as well. The efficiency of VoIP communication can be affected by the connection's quality as well as the addressing technology used. As a result, the effect of various bandwidths and addressing types was also studied.

\section{TOPOLOGY CREATION AND CONFIGURA- TION}

The first step was to design the interconnection of all devices so that their subsequent configuration did not require a change of interconnection. Therefore, it was necessary to specify which configuration would be used and how the network would be monitored during the design phase. The connection of terminal devices in terminal networks was attained using a switch. However, the condition for its selection was the PoE (Power over Ethernet) function, which allows the connection of VoIP phones without an additional power supply. The Cisco Catalyst 2960 switch used met these conditions. Two conditions were crucial for selecting a router: support for VoIP by the device itself in addition to the device's operating system. Finally, the Cisco 2811 router was selected, on which the c2800nmadvipservicesk 9 operating system including VoIP support was installed. [2] The Cisco IP Phone 7961 was used as the terminal device. An additional switch was built to connect the two routers since it was essential for the study to track connectivity through the entire network. Monitored communication from both local networks was also brought to this switch and finally intercepted using the Wireshark program.

The constitution of the proposed network's basic setup consists of setting basic parameters like IP and network cover, as well as building a VLAN to coordinate computers. The creation of the TFTP server provided a tool for updating IP phones and also a way to adapt them to use the SCCP protocol. The inclusion of the DHPC server in the topology 
ensured the automatic management of the IP addresses of the end devices connected to the network. VLAN managed these IP addresses to separate IP phones from other network devices. Static routing was chosen for the communication of two remote networks, as it was a small network and the goal was to eliminate fluctuations in network quality as much as possible.

In the more advanced part of the configuration, the QoS service was applied, which ensured at least the minimum operating conditions of VoIP communication during full network utilization. A filter caught SCCP and RTP packets, which are the core communication components of VoIP, to distinguish VoIP packets. SCCP configuration support provided the used routers. The configuration included setting up a VoIP gateway that stored: [2]

1. data on currently active phones and their settings

2. establishing a line and IP phone setup

3. defining the phone numbers that will allocate to it

One of the last major configuration tasks was the creation of dial-peer routing of VoIP communication. The dialpeer function enables VoIP connections even outside the local network. This is due to the assignment of a telephone number pattern that is associated with the IP address of the destination network. Thanks to this connection, communication will happen between VoIP gateways, which, after good contact, will trigger a VoIP session. The SCCP protocol manual provides more detail about the setup. [8]

\section{DESIGN OF EXPERIMENTS}

However, such a network would not provide any relevant data for the investigation. The network is occupied in regular communication not only by the examined VoIP communication but also by other network users' communication. A packet generator that generated the required network load replaced this communication. The NetScanTools Pro application was chosen as the generator, which contains amount settings and network monitoring options. "Packet Flooder" is a part of this program that allowed you to set the packet size, the interval between packets, the destination of the packet, and many other settings. If the generator was only used in one of the end networks, the network would suffer significantly and surveillance would be tainted. As a result, both end networks had packet generators installed, and the load was forwarded to each other.

The experiments were inspired by the current offer of ISPs in the area. There were three key bandwidths selected from this proposal as being the most often used by everyday people. For this reason, the focus was also not on the offer of internet via optic fiber, but on the slightly older VDLS technology. Bandwidths relevant to the research were 15.30 and $60 \mathrm{Mbps}$. The goal was to test how bandwidth affects the quality of VoIP communication at these bandwidths. This network's restriction applied the switch that connected the LAN routers. Another aim, as mentioned before, was to determine the benefits and drawbacks of the usage of various IP protocols and their implementation. The investigated implementations were IPv4, IPv4 with NAT translation, and
IPv6 protocol. These attempts were to discover how the implemented implementation of the IP protocol will affect service efficiency.

The first experiment compared the efficiency of VoIP information transmission to that of video stream transmission. The VLC media player tool, which allows streaming over a local network, was used to build the video stream. [5] For this reason, the whole experiment focused only on comparing different implementations of IP protocols, as we only change the bandwidth outside the local network. The created video stream uses RTP packets besides VoIP communication for transmission, so monitoring strategy and evaluating communication's quality is the same. [6] The ten times experiment replication used the same setup to acquire statistical checkers. Data capture took 30-35 seconds of the video stream as well as VoIP call. Created video stream was using a video loop. An audio track played during communication, which represented human speech, was used to simulate a VoIP call.

The second attempt looked into the impact of the IP protocol on VoIP communication efficiency as bandwidth varied. These efforts were made outside of the local network, making it more difficult to intercept this contact. To fully catch the entire touch, the communication had to be merged into a single location on the network, after which the monitor port was used. This function allows you to duplicate contact and forward it to the selected port. In this case, the duplication of monitored ports also submitted their communication to a separate device on which complete network monitoring took place. In this way, there was a possibility to monitoring contact from the moment the IP telephone and VoIP gateway were linked to the moment one of the users terminated the call. In this experiment, speech communication was also simulated by playing an audio track, as just creating VoIP communication without voice data transmission would have no informative value. As in the previous experiment, it is necessary to repeat the experiment at least ten times for each combination of IP protocol and bandwidth to obtain relevant data.

\section{EXPERIMENTAL RESULTS}

In this case, the Wireshark program did not only serve to intercept communication during the tests. Thanks to the built-in mechanisms for packet analysis, it enabled and simplified the evaluation of every communication that we archived after the experiments. The four most fundamental criteria that influence the quality of VoIP or video stream communication became the subject of each communication's investigation. [3] Packet loss is the most crucial connectivity parameter directly related to the quality and reliability of the network. The second monitored parameter is Max Delta, which indicates the time that separates the previous packet in the stream and the current packet. The last two observed parameters Max Jitter and Mean Jitter are directly related. The monitored communication is based on the regular creation and sending of packets, which is not necessarily the case. The Jitter parameter indicates the time difference between the expected time interval between packets and their real-time interval. These delays caused 
the network use and need compensation.

\subsection{Comparison of VoIP and video stream}

The graphs below show the results of a comparison of VoIP and video stream quality. The first graph shows the loss of packets during communication. Video streaming using the IPv4 protocol shows significantly lower packet loss than all other implementations in this experiment. The Max Delta graph shows significantly fewer video stream delays than VoIP. On the contrary, the graphs showing Jitter show a considerable advantage of VoIP communication, as the deviations between individual packets were minimal.

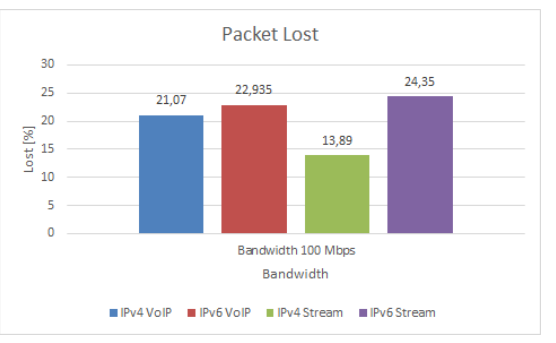

Fig. 2 Comparation of Packet Lost between VoIP and videostream.

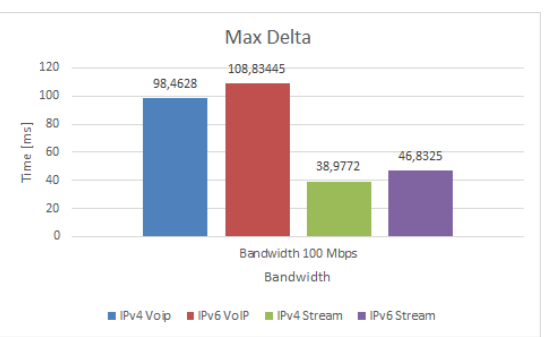

Fig. 3 Comparation of Max Delta between VoIP and videostream.

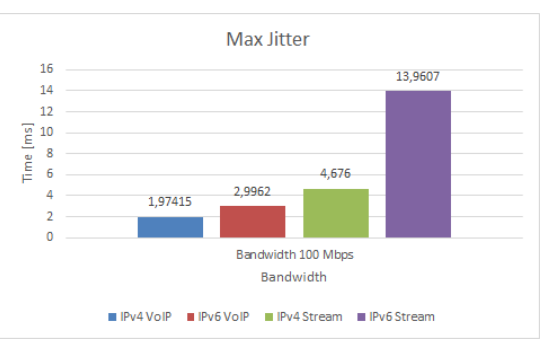

Fig. 4 Comparation of Max Jitter between VoIP and videostream.

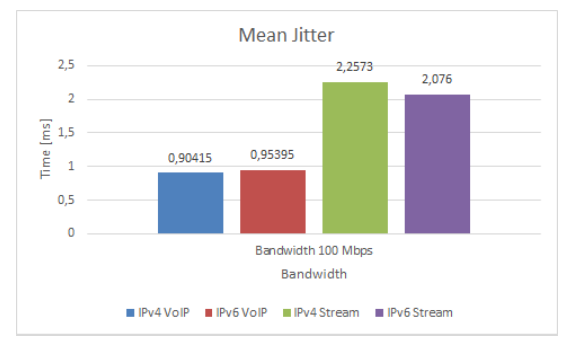

Fig. 5 Comparation of Mean Delta between VoIP and videostream.

\subsection{Comparison of VoIP at different bandwidths}

This experiment focused on the influence of bandwidth and the type of IP protocol implementation in the network. A necessary change was the size of the network load during the individual attempts to modify the bandwidth. For this reason, it is not possible to numerically compare the analyzed values between different bandwidths. Nevertheless, it is possible to conclude from the following graphs about the effect of bandwidth and the used implementation of the IP protocol on the quality of VoIP communication.

The first group of graphs is the results of an experiment at a bandwidth of 60 Mbps. IPv6 has the lowest packet loss, and IPv4 has only slightly worse results. In terms of maximum response, IPv4 has the best results. Both of these comparisons indicate that the IPv4 protocol with NAT implementation is the worst. The last graphs show the jitter of the monitored communication. In this case, IPv4 and IPv4 with NAT implemented are significantly better, and IPv6 appears to be the worst. [4]

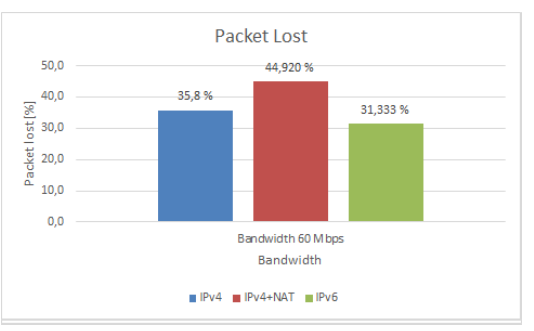

Fig. 6 Comparation of Packet Lost between different IP protocols using $60 \mathrm{Mbps}$ bandwidth.

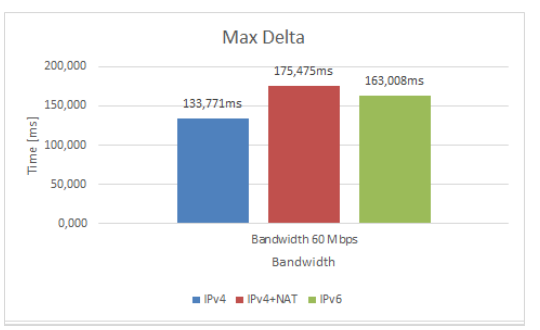

Fig. 7 Comparation of Max Delta between different IP protocols using $60 \mathrm{Mbps}$ bandwidth.

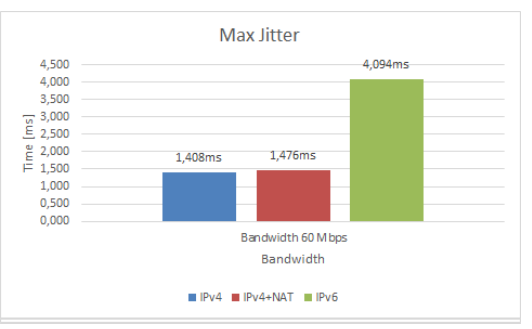

Fig. 8 Comparation of Max Jitter between different IP protocols using $60 \mathrm{Mbps}$ bandwidth. 


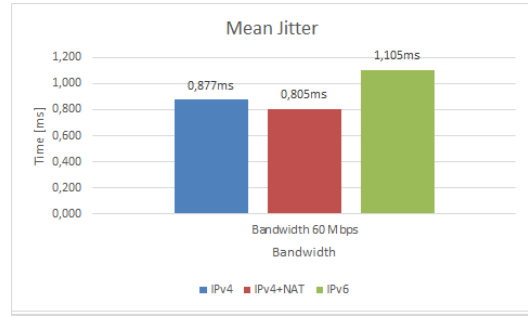

Fig. 9 Comparation of Mean Jitter between different IP protocols using $60 \mathrm{Mbps}$ bandwidth.

The second group of graphs shows the experiment at a bandwidth of $30 \mathrm{Mbps}$. The diagrams describing the loss of packets and the maximum response differ from the previous ones only by their numerical values. Generally, the result is the same, and therefore IPv4 with NAT appears to be the worst choice based on this comparison. However, the jitter of VoIP communication at a bandwidth of $30 \mathrm{Mbps}$ shows IPv4 with NAT as the best choice, so the spacing between packets is a lot less, and there are no packet outages. In this comparison, it again appears to be the worst IPv6 protocol.

The last group of graphs belongs to the experiment at a bandwidth of $15 \mathrm{Mbps}$. As in the previous cases, the IPv6 protocol has the very least packet loss, and the IPv4 protocol has the very least delay. IPv6 is again the least reliable protocol in terms of jitter. In this case, IPv4 seems to be the best protocol.

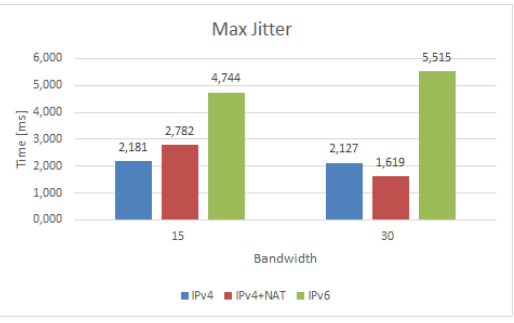

Fig. 10 Comparation of Max Jitter between different IP protocols using 15 and $30 \mathrm{Mbps}$ bandwidth.

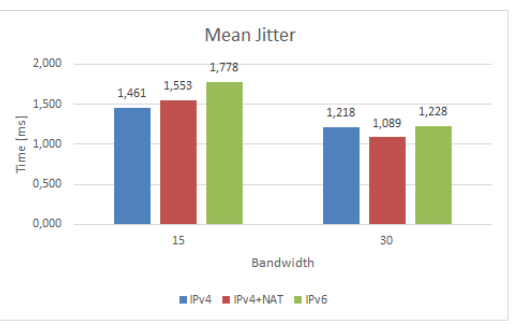

Fig. 11 Comparation of Mean Jitter between different IP protocols using 15 and $30 \mathrm{Mbps}$ bandwidth.

\section{CONCLUSION}

Based on the above results of the first attempt, it is clear that the video stream using the IPv4 protocol is of better quality. The reason is that it has the very least packet losses and also the minimal response from all compared communications. Since jitter is much less stable than a video stream, VoIP connectivity is less vulnerable to net- work transmission. The IPv6 protocol seems to be the better alternative in terms of failure in the second experiment, based on its results. IPv6 delays are also not the worst, but IPv4 has proven that it can be processed faster than IPv6. In terms of jitter, however, IPv6 has the worst results. Therefore the use of the IPv4 protocol would be a better choice for VoIP communication. Although the implementing results of the IPv4 protocol using NAT were the worst at all bandwidths, the jitter results suggest a possible advantage of this implementation at some bandwidths, such as $30 \mathrm{Mbps}$ bandwidth.

\section{ACKNOWLEDGEMENTS}

This research was supported by the Scientific Grant Agency of the Ministry of Education, Science, Research and Sport of Slovakia under the contract 1/0268/19.

\section{REFERENCES}

[1] ZIMERMANN, H.: OSI reference model-the ISO model of architecture for open systems interconnection. IEEE Transactions on communications 28.4 (1980): 425-432.

[2] BARZ, H. W. - BASSET G. A.: Other Standard VoIP Protocols." (2016): 183-212.

[3] IRWIN, D. - SLAY, J.: Extracting evidence related to VoIP calls. InIFIP International Conference on Digital Forensics 2011 Jan 31 (pp. 221-228). Springer, Berlin, Heidelberg.

[4] DAS, R. - TUNA, G.: Packet tracing and analysis of network cameras with Wireshark. In2017 5th International Symposium on Digital Forensic and Security (ISDFS) 2017 Apr 26 (pp. 1-6). IEEE.

[5] SUBEDI, P. - HONG, X.: Streaming Video Identification for Caching and Sharing. InProceedings of the SouthEast Conference 2017 Apr 13 (pp. 239-242).

[6] SCHULZRINNE, H. - CASNER, S. - FREDERICK, R. - JACOBSON, V.: Real-time transport protocol. RFC1899. 2003 Jul:21.

[7] DAWOOD, H.: IPv6 security vulnerabilities. International Journal of Information Security Science. 2012 Dec 29;1(4):100-5.

[8] LIANG, YC. - LEON, WS. - ZENG, Y. - XU, C.: Design of cyclic delay diversity for single carrier cyclic prefix (SCCP) transmissions with block-iterative GDFE (BI-GDFE) receiver. IEEE transactions on wireless communications. 2008 Feb 15;7(2):677-84.

Received March 29, 2021, accepted May 28, 2021

\section{BIOGRAPHIES}

Cyril Filip Kováčik is a student at the Department of Electronics and Multimedia Communications, Faculty of Electrical Engineering and Informatics, Technical University of Koice. His research interests include data analysis and modelling network infrastructures, theory and simulation of complex systems. 
Electrical Engineering and Informatics, Technical Univer-

Gabriel Bugár is a Research Assistant at the Department of Electronics and Multimedia Communications, Faculty of sity of Kosice, Slovakia. His research interests include cognitive networks, image processing, security and e-learning. 